\title{
Light Curves and Metal Abundances of RR Lyrae Variables in the Bar of the Large Magellanic Cloud
}

\author{
G. Clementini, A. Bragaglia, L. Di Fabrizio \\ Osservatorio Astronomico di Bologna, Via Ranzani 1, I-40127 Bologna, \\ Italy \\ E. Carretta, R. G. Gratton \\ Osservatorio Astronomico di Padova, Vicolo dell'Osservatorio 5, \\ I-35122 Padova, Italy
}

\begin{abstract}
The Large Magellanic Cloud (LMC) is widely considered a corner-stone of the astronomical distance scale. However, a difference of $0.2-0.3 \mathrm{mag}$ exists in its distance as predicted by the short and long distance scales. Distances to the LMC from Population II objects are founded on the RR Lyrae variables. We have undertaken an observational campaign devoted to the definition of the average apparent luminosity, and to the study of the mass-metallicity relation for RR Lyrae stars in the bar of the LMC. These are compared with analogous quantities for cluster RR Lyrae stars. The purpose is to see whether an intrinsic difference in luminosity, possibly due to a difference in mass, might exist between field and cluster RR Lyrae stars, which could be responsible for the well-known dichotomy between short and long distance scales. Preliminary results are presented on the $V$ and $B-V$ light curves, the average apparent visual magnitude, and the pulsational properties of 102 RR Lyrae stars in the bar of the LMC, observed at ESO in January 1999. The photometric data are accurately tied to the Johnson photometric system. Comparison is presented with the photometry of RR Lyrae stars in the bar of the LMC obtained by the MACHO collaboration (Alcock et al. 1996). Our sample includes 9 double-mode RR Lyrae stars selected from Alcock et al. (1997) for which an estimate of the metal abundance from the $\Delta S$ method is presented.
\end{abstract}

\section{Observations, Data Reduction, and Calibration}

We have collected $B, V$ CCD photometry in two $13^{\prime} \times 13^{\prime}$ fields located close to the bar of the LMC and overlapping with fields \#6 and \#13 of the MACHO microlensing experiment (see http://wwwmacho.mcmaster.ca), using the 1.5-m Danish telescope in La Silla. The photometric dataset consists of $58 \mathrm{~V}$ and 25 $B$ frames of each field. 118 variables were identified in the two fields (62 RRab, $30 \mathrm{RRc}, 10 \mathrm{RRd}$ stars, 6 Cepheids, 9 eclipsing binaries and $1 \delta$ Scuti star). Photometry was accurately tied to the Johnson standard photometric system using a large number of standard stars from Landolt (1992). 

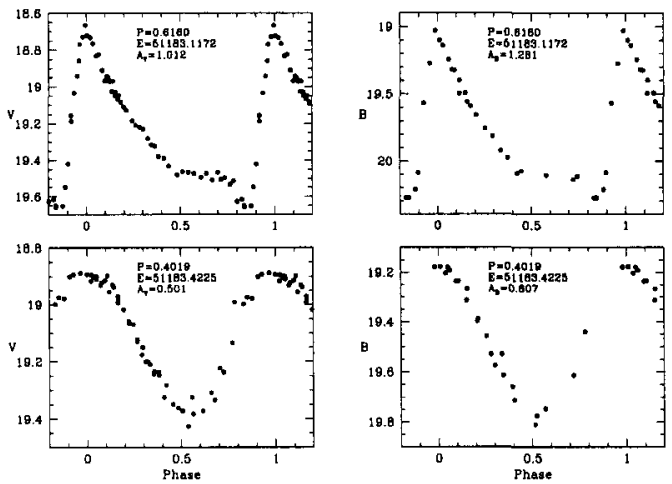

Figure 1. $\quad V$ and $B$ light curves of RR Lyrae variables in our sample.

Low resolution spectra $(R=450$, res. element $=9 \AA)$ were obtained for 7 of the RRd variables with EFOSC2 at the 3.6-m ESO telescope, and metal abundances have been derived using the $\Delta S$ technique (Preston 1959). For calibration purposes we took also spectra at minimum light of 8 field RR Lyrae stars of known $\Delta S$ (including an RRc followed along the pulsation cycle), and of 14 stars of the open cluster Collinder 140, which contains spectral type standard stars.

Photometric data were analyzed using the package DoPHOT (Schechter, Mateo, \& Saha, 1993). Spectroscopic data were reduced using the standard IRAF packages for long slit spectra. Total numbers of 28000 and 25000 , and 23000 and 19000 objects were measured in the $V$ and $B$ frames of the two fields, respectively. The average magnitude the LMC clump stars is $\langle V\rangle=19.202$ $(\sigma=0.202 ; 8979$ stars $)$. The comparison with the Alcock et al. (1997, hereinafter A97) $\langle V\rangle$ for the clump, as can be read from their Fig. 3, shows that our value is about $0.10 \mathrm{mag}$ "fainter".

\section{Identification, Period Search and Pulsational Properties of the RR Lyrae Variables}

Variables were identified on the $V$ and $B$ frames independently. Periods were defined using the program GRATIS (GRaphical Analyzer TIme Series; Montegriffo, Clementini, \& Di Fabrizio, in preparation) which was run on the differential photometry of the variables with respect to a number of stable reference stars. The period search procedure was to perform a Lomb analysis (Lomb 1976) on a wide period interval first, and then use a Fourier analysis to refine periods and find the best fitting models. Average residuals from the best fitting models for single-mode pulsators with well sampled light curves are $0.02-0.03 \mathrm{mag}$ in $V$, and $0.04-0.06 \mathrm{mag}$ in $B$. Fig. 1 shows examples of the $V$ and $B$ light curves of an RRab and an RRc in our sample. The period distribution of the RRc stars in our fields peaks at $\langle P(\mathrm{RRc})\rangle=0.314 \pm 0.047 \mathrm{~d}$ (average of 30 stars), while $\langle P($ RRab $)\rangle=0.577 \pm 0.077 \mathrm{~d}$ (average of 60 stars) to compare with $0.342 \mathrm{~d}$ and $0.583 \mathrm{~d}$ of Alcock et al. (1996, hereinafter A96). Our shortest period RRab has period $0.318 \mathrm{~d}$, and there are two other RRab stars with periods around 
$0.40 \mathrm{~d}$. We derived $\langle V\rangle$ and $\langle B\rangle$ intensity-averaged magnitudes, as well as $V$ and $B$ amplitudes $\left(A_{V}\right.$ and $\left.A_{B}\right)$ for all variables with complete light curves. The average $\langle V\rangle$ apparent magnitude of the RR Lyrae stars in our sample is : $\langle V\rangle=19.325 \pm 0.170$ (75 stars), to compare with $\langle V\rangle=19.4$ from A96. On the assumption that: $E(B-V)=0.10$ (Bessell 1991) and $A_{V}=3.1[E(B-V)]$ for the $\mathrm{LMC}$ we find:

$\left\langle V_{0}\right\rangle=19.015 \pm 0.020$ at $[\mathrm{Fe} / \mathrm{H}] \sim-1.5$ field $\mathrm{RR}$ Lyraes, this paper

$\left\langle V_{0}\right\rangle=19.09$ at $[\mathrm{Fe} / \mathrm{H}] \sim-1.7$ field $\mathrm{RR}$ Lyraes, A96

$\left\langle V_{0}\right\rangle=19.06 \pm 0.06$ field RR Lyraes, Kinman et al. (1991)

$\left\langle V_{0}\right\rangle=18.94 \pm 0.040$ at $[\mathrm{Fe} / \mathrm{H}]=-1.9$ cluster $\mathrm{RR}$ Lyraes, Walker (1992)

Allowing for the 0.4 dex difference in $[\mathrm{Fe} / \mathrm{H}]$, our $\left\langle V_{0}\right\rangle$ is in very good agreement with Walker (1992), thus showing that there is no clear evidence for a difference in luminosity between field and cluster RR Lyrae stars in the LMC.

Table 1. $\Delta S$ values, metal abundance and masses of RRd pulsators

\begin{tabular}{cclc}
\hline No.(A97) & $\Delta S$ & {$[\mathrm{Fe} / \mathrm{H}]$} & $M / M_{\odot}$ \\
\hline 2 & 8.6 & -1.74 & 0.60 \\
5 & $11.3:$ & $-2.28:$ & 0.61 \\
45 & 7.9 & -1.62 & 0.65 \\
48 & 5.2 & -1.09 & 0.67 \\
49 & 7.3 & -1.50 & 0.69 \\
61 & 5.9 & -1.23 & 0.71 \\
67 & 8.8 & -1.78 & 0.81 \\
\hline
\end{tabular}

\section{Spectroscopy of the Double-Mode RR Lyrae Stars}

Spectra for 7 of the RRd variables falling in our fields were obtained at phases corresponding to the minimum light. Metallicities were inferred from these spectra using the $\Delta S$ index and the Clementini et al. (1995) calibration of $\Delta \mathrm{S}$ in terms of metallicity $([\mathrm{Fe} / \mathrm{H}]=-0.194 \times \Delta \mathrm{S}-0.08)$. Dealing with variables which pulsate both in the fundamental and first overtone, the question arises whether these stars should be treated as RRab or RRc pulsators in measuring $\Delta \mathrm{S}$. Following Kemper (1982) and discussions in Clement, Kinman, \& Suntzeff (1991), we considered our targets as RRc variables. $\Delta S$ values were thus measured from spectra with Hydrogen spectral type later than A8, applying phase corrections derived from the field $R R c T$ Sex. Table 1 lists the $\Delta S$ values and corresponding metallicities we derived for our targets. Values for star \#5 are rather uncertain since the spectrum of this star has very low $S / N$. Errors on the quoted $\Delta S$ are of the order of $0.7-1 \Delta S$ subclasses, corresponding to an error of about $0.20-0.30$ $\operatorname{dex}$ in $[\mathrm{Fe} / \mathrm{H}]$.

\section{The Mass-Metallicity Relation for the RR Lyrae Stars}

A97 provides $P_{0} / P_{1}$ ratios for the 7 double-mode variables in Table 1 . These ratios can be used together with Petersen diagrams (Petersen 1973) and Bono et al. (1996) loci of model pulsators to estimate the masses of our targets (see, 
e.g., Fig. 2 of A97). Masses obtained with this procedure are listed in the last column of Table 1 and plotted against metallicity in Fig. 2. Also shown is the mass-metallicity relation defined by double-mode pulsators in the globular clusters M68 (Walker 1994), M15 (Nemec 1985) and IC 4499 (Clement et al. 1986; Walker \& Nemec 1996) and two RRd stars in the Milky Way (Clement et al., 1991). Although there is some scatter, and there are only few field objects, most of the LMC RRd stars seem to follow the general mass-metallicity relation defined by the cluster RRd stars. Hence, no clear-cut evidence is found for a difference in mass between field and cluster RR Lyrae stars.

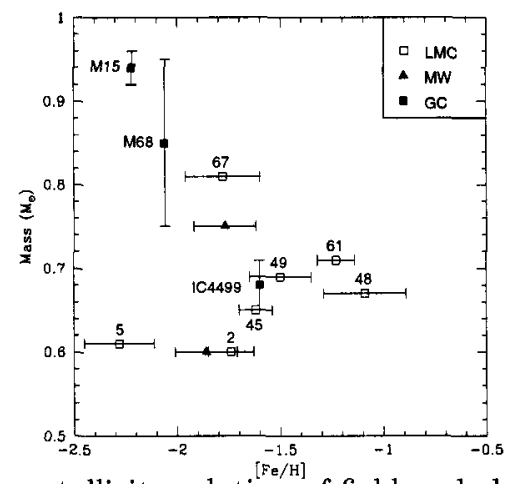

Figure 2. Mass-metallicity relation of field and cluster double-mode pulsators

\section{References}

Alcock, C., Allsman, R. A., Axelrod, T. S., et al. 1996, AJ, 111, 1146 [A96]

Alcock, C., Allsman, R. A., Alves, D., et al. 1997, ApJ, 482, 89 [A97]

Bessell, M. S. 1991, A\&A, 242, L17

Bono, G., Caputo, F., Castellani, V., \& Marconi, M. 1996, ApJ, 471, L33

Clement, C. M., Nemec, J. M., Robert, N., et al. 1986, AJ, 92, 825

Clement, C. M., Kinman, T. D., \& Suntzeff, N. B. 1991, ApJ, 372, 273

Clementini, G., Carretta, E., Gratton, R. G., et al. 1995, AJ, 110, 2319

hemper, E. 1982, AJ, 87, 1395

Kinman, T. D., Stryker, L. L., Hesser, J. E., et al. 1991, PASP, 103, 1279

Landolt, A. 1992, AJ, 104, 340

Lomb, N. R. 1976, Ap\&SS, 39, 447

Nemec, J. M. 1985, AJ, 90, 240

Petersen, J. O. 1973, A\&A, 27, 89

Preston, G. W. 1959, ApJ, 130, 507

Schechter, P. L., Mateo, M., \& Saha, A. 1993, PASP, 105, 1342

Walker, A. R. 1992, ApJ. 390, L81

Walker. A. R. 1994, AJ. 108, 555

Walker, A. R. \& Nemec. J. M. 1996, AJ. 112, 2026 